EOMmUn Communication et organisation

Dम매I 32 | 2007

La ville dans tous les sens

\title{
Entretien avec Claude Lévêque
}

Marie-Pascale Mignot

\section{OpenEdition}

Journals

Édition électronique

URL : http://journals.openedition.org/communicationorganisation/402

DOI : 10.4000/communicationorganisation.402

ISSN : $1775-3546$

\section{Éditeur}

Presses universitaires de Bordeaux

Édition imprimée

Date de publication : 1 décembre 2007

Pagination : 225-233

ISSN : 1168-5549

\section{Référence électronique}

Marie-Pascale Mignot, « Entretien avec Claude Lévêque », Communication et organisation [En ligne], 32

2007, mis en ligne le 01 décembre 2010, consulté le 21 septembre 2020. URL: http://

journals.openedition.org/communicationorganisation/402 ; DOI : https://doi.org/10.4000/

communicationorganisation. 402

(c) Presses universitaires de Bordeaux 


\section{Entretien avec Claude Lévêque par Marie-Pascale Mignot}

architecte à Bordeaux', qui a collaboré, en 2007, avec Claude Lévêque sur le projet de maison de services pour Réseau 32.

Claude Lévêque ${ }^{2}$ est un artiste plasticien français, présent sur la scène de l'art contemporain.

Né en 1953 à Nevers, il vit et travaille à Montreuil en Région parisienne, à Pèteloup dans la Nièvre et partout ailleurs. Après des études à l'école des Beaux-Arts de Bourges, il démarrera son parcours d'artiste dans les années quatre-vingt. C'est à Paris, qu'il côtoiera le monde de la publicité, de la musique et de l'art contemporain. Il deviendra un producteur d'installations qui ne se répètent pas car ce sont les « aspects relationnels et les vibrations d'un lieu » qui guident son travail.

Attiré par les lieux singuliers, Claude Lévêque joue avec eux dans l'espace public ou les travaillent de l'intérieur. Il aime "placer les visiteurs en embuscade » pour déjouer le rationnel et inventer une sensorialité calculée. Il transforme les lieux avec la lumière et le son pour construire des installations renversantes et décalées qui amènent le questionnement sur soi et les autres. L'espace, qu'il investit, est libre d'interprétations, de manipulations, d'expérimentations pour permettre un croisement de son histoire avec le lieu et les hommes. Il cherche à ce que le visiteur se positionne.

Ces installations, stimulées par l'appropriation des visiteurs, sont des invitations au voyage dans un univers poétique et sensible en contrepoint du « monde dur et impitoyable d'aujourd'hui $»^{3}$.

\section{Euvres et installations}

\section{7}

Barcelone, La guerre du chocolat, $\mathrm{KBB}$

Rome, Le rôdeur, Luce di Pietra, Galerie des Carracci, Palais Farnèse

\footnotetext{
${ }^{1}$ Marie-Pascale Mignot est aussi Maître de conférence associée à l'ISIC (Institut des Sciences de l'Information et de la Communication) de l'Université Bordeaux 3 et coresponsable du master Stratégies et produits de communication.

${ }^{2}$ Troncy E.; Claude Lévêque, collection " Monographies d'artistes contemporains ", Paris, Éditions Hazan, 2001 - 112 pages, 100 illustrations

${ }^{3}$ Favier Christian, in catalogue d'exposition Claude Lévêque - Musée d'Art Contemporain du Val-de-Marne - édition MAC VAL - 2006
} 


\section{Entretien}

Albi, Le crépuscule du jaguar, Moulins Albigeois

Paris, Mon repos aux Tuileries, Fiac 2007

Miami, Scarface, Art Fair Basel, Galerie Kamel Mennour

Paris, Welcome to suicide park, Galerie Kamel Mennour

\section{En projet ou en exposition}

\section{8}

Uckange, (Lorraine) Tous les soleils, Haut Fourneau U4, MultiVisions nocturnes à partir du $1 \mathrm{er}$ mai

Saint-Louis (Haut-Rhin) (Valérie Jouve, Philippe Cognée, Claude Lévêque), Musée Fernet Branca : 1er juin à novembre

Toulouse : Printemps de septembre

Paris : Correspondance, Musée d'Orsay

\section{9}

San Paulo, Le grand sommeil

\section{0}

Russie : Les années de la France en Russie et de la Russie en France

Paris: Scénographie sur chorégraphie d'Angelin Preljoka d'après Siddhartha Hesse - Opéra Bastille.

Claude Lévêque, votre travail est métaphorique, il est lié à la sensibilité et à l'affect. Comment construisez-vous l'interaction entre vos ouvres et les lieux dans lesquels vous intervenez?

Le lieu sert de support à la construction de l'œuvre, à l'élaboration du langage artistique. A partir de l'histoire du lieu, de sa fonction, de ce qui s'y passe, je repère dans le site l'aspect technique, les sensations que le lieu me procure. Je rassemble tous ces éléments : photos, plans, schémas qui servent de point de départ à mon travail. Ce qui compte, ce sont les anecdotes que les gens me racontent sur l'endroit et les évocations que l'espace suscite en moi. Je vais m'attacher à ces univers, leur passé, leur présent. A partir de tous ces éléments que je transforme, je mets en place une fiction: une sorte de scénario d'éléments sensoriels, de lumière, de sons, de textures, de reflets avec différentes affectations qui peuvent venir de l'actualité, de ce qui se passe dans le monde. Plusieurs éléments peuvent se croiser et construire un nouveau langage. Je dis souvent que j'ai beaucoup de plaisir à travailler in situ sur les lieux, chacun étant différent. Chaque intervention est spécifique, elle s'adapte au lieu, même si il y a un lien général entre mes œuvres. Chaque œuvre est singulière et s'appuie sur une technique qui lui est propre. En 2003, j'ai fait une installation à 
l'école d'architecture de Grenoble Manifesto . Elle est assez légère et joue sur la fréquentation des lieux, les passages des étudiants et du personnel dans l'école. Je suis resté au plus près de cette situation : un croisement de lieux communs du langage utilisés dans les couloirs ou dans les passages. A partir d'une difficulté de lecture de l'espace architectural, j'ai décidé de faire un détour du côté des occupants avec des mots qu'ils ont choisis, et que j'ai placés dans des endroits du bâtiment pour les mettre en dialogue. Certaines évocations sont devenues des formes (escalier néon, construction briques).

L'intervention sur le Haut fourneau d'Uckange ${ }^{4}$ Tous les soleils en Lorraine joue sur la réactivation du lieu comme une espèce d'embryon où s'y redéveloppe des tas de choses, dans tous les sens. J'ai imaginé un nouveau parcours à travers des plateformes, passerelles, coursives et cheminements dans un site industriel en friche. Le public peut se promener dans l'œuvre où lumière et son participent à sa construction.

Le projet La maison où j'ai grandi à Plougonver, dans le village dans les Côtes d'Armor est une réflexion sur le lieu de mémoire. Je suis intervenu sur une maison qui accueillait, jusque dans les années 90, des personnes en difficulté. J'ai recueilli des anecdotes sur cette maison où les gens du village apportaient des vivres, du bois pour se chauffer et se retrouvaient. J'ai installé les structures évidées d'une table et 4 chaises apparaissant en lévitation avec des lampes projetant leurs silhouettes fantomatiques sur les murs. J'ai remplacé le toit existant par un caisson de vitrage et posé deux escaliers, en diagonale sur les murs extérieurs permettant d'observer au dessus du toit, en vue plongeante et croisée, l'intérieur de la maison où j'ai grandi.

Le projet Vie en chemin pour Réseau $32^{5}$ à Bordeaux, est une autre situation par rapport au lieu. Je trouve pas mal aussi de se confronter à la réalité de fonction d'un bâtiment. J'ai travaillé sur les besoins du lieu, en termes d'équipements, de lumière plus que sur les éléments formels. L'immeuble Paul Bert, dans le centre historique, n'était pas l'endroit où l'on emmène des éléments de contemplation d'ordre artistique. C'est un immeuble à vivre, dans lequel je peux détourner la réalité de fonctionnement, les besoins de lumière, les besoins du quotidien pour l'hygiène corporelle. On va utiliser ce qui se passe dans un tel équipement, la fréquentation des personnes, leurs besoins

\footnotetext{
${ }^{4}$ Parc du Haut Fourneau U4 à Uckange - Communauté d'Agglomération du Val de Fensch - 2007

${ }^{5}$ Maison de services aux personnes en précarité, avec une laverie solidaire, un espace hygiène avec hammam, un bar-brasserie avec espace cyber, architecte de la réhabilitation de l'immeuble : Marie-Pascale Mignot - 2007
} 


\section{Entretien}

et puis en faire quelque chose par rapport aux fonctions que l'on propose. Cela m'intéresse beaucoup. Il s'en dégage un univers poétique où je ne me sens pas frustré.

Pour le parcours du nouveau tramway T3 des Maréchaux Sud, aux portes de Paris, des artistes ${ }^{6}$ ont été invités à imaginer des ouvres en relation avec le projet urbain d'aménagement. Les propositions devaient être en relation étroite avec la ville. Vous avez choisi une installation " Tchaïkovski " qui semble jouer avec l'environnement sensoriel, avec les ambiances. Quelle est la part dans cette ouvre de l'architecture, du contexte urbain et de la mobilité?

Dans mon intervention sur le tramway, l'œuvre est simple à décrypter, car très visuelle. J'ai travaillé sur la façade discrète d'une petite construction. C'est une simple vanne d'alimentation en eau de la Ville de Paris. Le bâtiment est un parallélépipède que je couronne d'une sorte de diadème en inox brillant, aux surfaces martelées, évoquant les reliefs d'une surface d'eau qui vibre au vent. D'ailleurs, j'ai appelé ça « Tchaïkovski », pour introduire un lien symbolique amusé avec le lac des cygnes - un retour troublant sur la vie du musicien. Cela donne une impression d'eau, un reflet miroitant, réfléchissant. L'idée, c'est de représenter l'eau, à partir de panneaux obliques posés sur le bâtiment à la manière de rabats d'un carton. Ils prennent la lumière selon leur inclinaison et reflètent tout ce qui se passe dans la rue, aussi bien le mouvement des voitures, que celui des passants ou celui du tramway. L'œuvre est un écran, un miroir de l'environnement extérieur à travers toutes ces métamorphoses. Le mouvement est important dans mon travail, il se répercute par le reflet ou l'idée du reflet, qui peut prendre en charge le passage d'un visiteur. A la symbolique de l'eau, s'ajoute la symbolique du temps qui passe avec tous les moments différents de la journée, les changements de climat et d'atmosphère. Les ambiances, les textures sont toutes différentes. L'inox se mélange au ciel. Le soir, les feux tricolores, les enseignes se reflètent dans le métal. Tout est scintillement. Il y a un jeu de mouvements intéressant. Le bâtiment du projet est installé entre deux rues parallèles, au cœur de la Cité universitaire internationale. En mauvais état, j'ai demandé sa restauration architecturale. Sur sa façade principale, deux petits médaillons qui crachent de l'eau, ont été mis en

\footnotetext{
${ }^{6}$ « $1 \mathrm{SQMH} »$ de Didier Fiuza Faustino, « Skate Park » de Peter Kogler, « Mirage » de Bertrand Lavier, «Murmures » de Christian Boltanski, «Tchaïkovsky » de Claude Lévêque, «Incubate Lactate Perambulate » d'Angela Bulloch, "Pont» de Peter Kogler, «From Boullée to Eternity » de Dan Graham, et « le téléphone » de Sophie Calle avec Frank Ghery
} 
valeur. Ils étaient complètement condamnés et je les ai fait remettre en service. Cette installation sur le tracé du tramway a une résonnance poétique.

\section{Quelle différence de sens accordez-vous entre l'exposition d'une ouvre dans l'espace du musée et l'installation in situ?}

Moi, j'aime bien les espaces temporaires, les situations temporelles. Je n'aime pas l'art figé. A l'idée qu'une œuvre d'art soit toujours au même endroit 20 ans après, cela me terrifie (chez moi, je ne stocke rien). Le musée fait vivre l'œuvre, c'est différent, elle est prise dans une classification patrimoniale, celui-ci à un rôle qui s'explique. D'autres types d'expériences font appel à une rencontre éphémère avec l'œuvre. Elles doivent avoir une résonnance par rapport à l'actualité, après elles peuvent disparaître ou se métamorphoser en autre chose. Le musée doit pouvoir faire durer une œuvre. Les interventions extérieures ont une durée plus discutable. Cependant, je ne donne de leçon à personne, c'est un point de vue personnel.

\section{Et l'art urbain?}

Je fais peu d'art urbain. Je ne me sentais pas du tout à l'aise pour intervenir dans des lieux extérieurs. J'étais plutôt confiné dans l'espace intérieur, je travaillais sur l'obscurité via la lumière. Cela me paraissait insurmontable d'intervenir dans le milieu urbain (j'avais mené plusieurs commandes publiques qui avaient échoué). Maintenant, ça m'intéresse. Je pensais que jamais je n'interviendrais à l'extérieur. Puis, il y a eu plusieurs propositions et notamment des commandes publiques qui m'ont intéressé. L'intervention artistique publique en extérieur n'est pas du tout la même que celle que l'on peut effectuer dans un musée ou dans un centre d'art. Je fais vraiment une différence, on ne cherche pas à faire quelque chose de consensuel ou de joli pour conquérir un maximum d'intérêt de la part des gens. On peut garder une certaine position, sans forcément dénaturer la chose pour la rendre plus accessible. On peut tout à fait garder ses positions, car je pense que l'art ou les projets publics sont des éléments imposés au public. En effet, lorsque les gens passent dans la ville pour aller travailler, par exemple, ils sont confrontés à l'œuvre sans avoir eu le choix. Ils ne sont donc pas dans la même situation que lorsqu'ils se rendent dans un musée ou dans un centre d'art. Il y a une différence entre le choix et l'obligation. Cela fait partie des contraintes urbaines. J'ai fait une intervention éphémère dans un centre commercial abandonné qui se trouve derrière la Cité des sciences de la Villette à Paris, en soulignant l'espace de manière extrêmement discrète avec une proposition pratiquement invisible. J'ai joué sur le 


\section{Entretien}

lieu pour être le moins démonstratif possible. La plupart des passants n'ont donc rien vu, en trouvant presque normale mon intervention. Je suis donc intervenu dans le milieu urbain, en faisant quelque chose d'assez radical, en étant à contre courant du sens qui pouvait être donné à l'espace. C'était une boutique qui avait vraiment le profil d'une caisse. Or, j'avais trouvé une caisse qui se rapprochait d'un modèle réduit de la vitrine de centre commercial et j'ai construit à l'intérieur une enceinte fermée contre les trois côtés de la vitrine : une chose qui était complètement en écho avec le lieu et avec un mouvement de lumière qui pourrait être comme une alerte, une palpitation lumineuse. La forme de l'installation était comme naturelle, comme une situation normale par rapport au lieu, pour les gens qui arrivaient du métro. Elle était invisible.

Quelle est la place de l'œuvre d'art dans la ville, face aux médias publicitaires et au design urbain?

Souvent, on ne voit pas l'œuvre d'art, elle a un langage tellement autre que celui du design urbain ou de la publicité, qui joue directement sur l'impact visuel et se met en situation de répondre au passage des gens. L'artiste peut aussi répondre au passage de l'homme dans la ville, mais c'est plus discret parce que moins utilitaire. La publicité est faite pour consommer car sa position a un impact extrêmement calculé. Le mobilier urbain, c'est tout ce qui sert à s'abriter, à attendre, il répond au besoin du stationnement et du passage. L'art, c'est autre chose, c'est un moment, un temps autre sur la perception. Cela peut être une interrogation sur le statut social du passant, cela peut refléter l'histoire $\mathrm{du}$ lieu, du quartier, de la zone d'activités dans sa symbolique et sa forme. C'est une réponse que l'on peut constater dans les œuvres d'art qui marquent différents types de lieux. Avec les Nouveaux Commanditaires ${ }^{7}, c^{\prime}$ 'est autre chose, la démarche est étroitement liée à des personnes implantées localement (municipalités, habitants d'une région) souhaitant qu'un artiste se confronte à un élément de leur

\footnotetext{
${ }^{7}$ Le programme Nouveaux Commanditaires de la Fondation de France est « une réponse possible pour les hommes et les femmes qui, en manque de repères réclament un réinvestissement du symbolique dans leur vie et leur environnement, et ne se suffisent plus de réponses techniques ou administratives aux problèmes qu'ils se posent. Cette action permet à de citoyens confrontés à des enjeux de société ou de développement d'un territoire, d'associer des artistes contemporains, de toutes disciplines, à leur préoccupation par le biais d'une commande .» Depuis plus de dix ans, les Nouveaux commanditaires ont été à l'origine de 175 commandes réalisées ou en cours avec des partenaires différents parmi lesquels les communes, aux côtés du ministère de la Culture et de différents autres services de l'Etat, des entreprises privées, de conseils généraux, des conseils régionaux et des associations.
} 
patrimoine. Un élément qu'ils souhaitent garder, en lui redonnant vie au travers d'une proposition artistique. Cette disposition est moins imposée à l'artiste, qu'une commande publique. C'est une histoire plus intime.

Pour vous, le lieu de l'art semble être partout, même dans les lieux qui semblent abandonnés. Comment travaillez-vous sur le tissu urbain celui des centres villes ou celui de la banlieue?

La banlieue, c'est un lieu de choc des cultures, des générations, des ethnies. Pour moi, c'est le lieu le plus vivant dans la ville. La plupart du temps, les centres villes sont morts, ce sont des cimetières ou des musées. Partout, à Bordeaux comme à Paris, dans toutes les grandes villes, les centres urbains sont des vitrines mortifères. C'est autour que ça bouge, qu'il y a de l'identité, de la réactivité, de la création. Mais ce n'est pas moi qui fais ce constat. Les villes sont devenues des bonbonnières : la restauration, c'est fait pour être plaisant, joli, pour arroser les pouvoirs politiques. Ce sont des vitrines, mais moi je n'y participe pas. Je ne sais pas si les villes tellement restaurées sont propices à des questionnements contemporains.

La banlieue, oui, c'est la confrontation avec des classes populaires, plus mélangées, avec des aspects très intéressants et pas que des situations de violences. Ça régénère tout! Une ville comme Paris, capitale de la France, pour moi c'est une ville finie, nous la connaissons par cœur, les touristes la magnifient. Alors qu'à l'extérieur, c'est complètement différent, dès que l'on sort y a des choses qui se passent, des choses dérangeantes qui font venir peu de touristes, mais la banlieue parisienne regorge d'architectures extraordinaires.

Quel sens accordez-vous à vos installations in situ ou aux dispositifs éphémères que vous mettez en scène dans les galeries d'art ou dans les musées?

C'est intéressant comme expérience, parce qu'on ne peut pas faire plus dans ces lieux-là, on ne peut rien imposer. Après, les choses peuvent êtres vues et interprétées de différentes manières, c'est ouvert à tout le monde. Je trouve que ce n'est pas le même impact que dans une galerie ou un musée, où il pourrait y avoir une relation plutôt proche dans l'idée d'un contact avec un langage ou une œuvre d'art.. A l'extérieur, c'est différent. Les gens passent et c'est ce qui m'intéresse. C'est ce phénomène du passage qui réduit la lecture de l'objet. Nous ne sommes plus dans la contemplation, il y aura des gens qui ne s'arrêteront jamais dans la ville. Ils feront peut être le choix 


\section{Entretien}

d'aller voir l'intervention d'un artiste dans le contexte urbain, mais beaucoup de passants ne la verront même pas. Si la réalisation est spectaculaire, c'est différent, c'est un autre choix de conception.

A travers cette expérience, avez-vous contribué à une nouvelle conception de la ville? Est-ce que le dispositif urbain qui intègre l'art, restitue une autre ville?

Je n'en sais rien, c'est difficile, il faut voir les choses après, à l'usage. Je n'aurais jamais la prétention de dire que j'ai changé la lecture de la ville. J'ai souvent été sceptique sur la commande publique, mais je pense que tout le monde y a un peu réfléchi aussi bien les commandes d'états que les Nouveaux Commanditaires. Si les Nouveaux Commanditaires existent aujourd'hui, c'est aussi parce qu'il y avait des questions à se poser sur le fait d'imposer l'art, avec le $1 \%$ d'art public associé à une construction ou à un aménagement urbain.

Il faut se questionner sur le type de commande publique qui installe une «statue de bronze» dans un lieu pour l'éternité. En effet, avec le temps un décalage s'installe entre l'œuvre et le contexte urbain, qui se métamorphose souvent selon les besoins d'aménagement. Si une œuvre d'art ou une intervention artistique a bien vécu et si les gens se la sont appropriée, elle fait partie d'une période, d'une époque de réalisation ou d'aménagement, c'est essentiel. Après, il faut que l'artiste accepte qu'elle soit enlevée, déplacée ou encore mise au musée. Pour le patrimoine ou un lieu à charge patrimoniale, c'est différent, car je pense que les choses peuvent être incluses dans le temps.

Ces thématiques urbaines révèlent des fragmentations de la ville et créent des ruptures. L'intervention artistique peut-elle créer du lien dans la ville déterritorialisée?

Ce n'est pas suffisant. Sur l'artiste pèsent des attentes de stabilité parce qu'il y a souvent des lacunes par ailleurs et que l'art reste un domaine irrationnel qui suscite une attente. Moi, je pense que l'art n'est pas suffisant pour changer la ville et on le sait bien. Mais il peut contribuer à créer des espaces de questionnement, de bien-être, de convivialité comme l'aménagement d'un terrain de boules, d'une piste de skate comme l'on fait de nombreux artistes contemporains. On peut également jouer sur des besoins de convivialité ou de fonctionnalité. Cela peut être un espace de méditation comme un jardin, des pauses dans la mobilité urbaine. Effectivement, l'artiste peut aider à créer du lien, mais cela ne va pas changer la vie. 

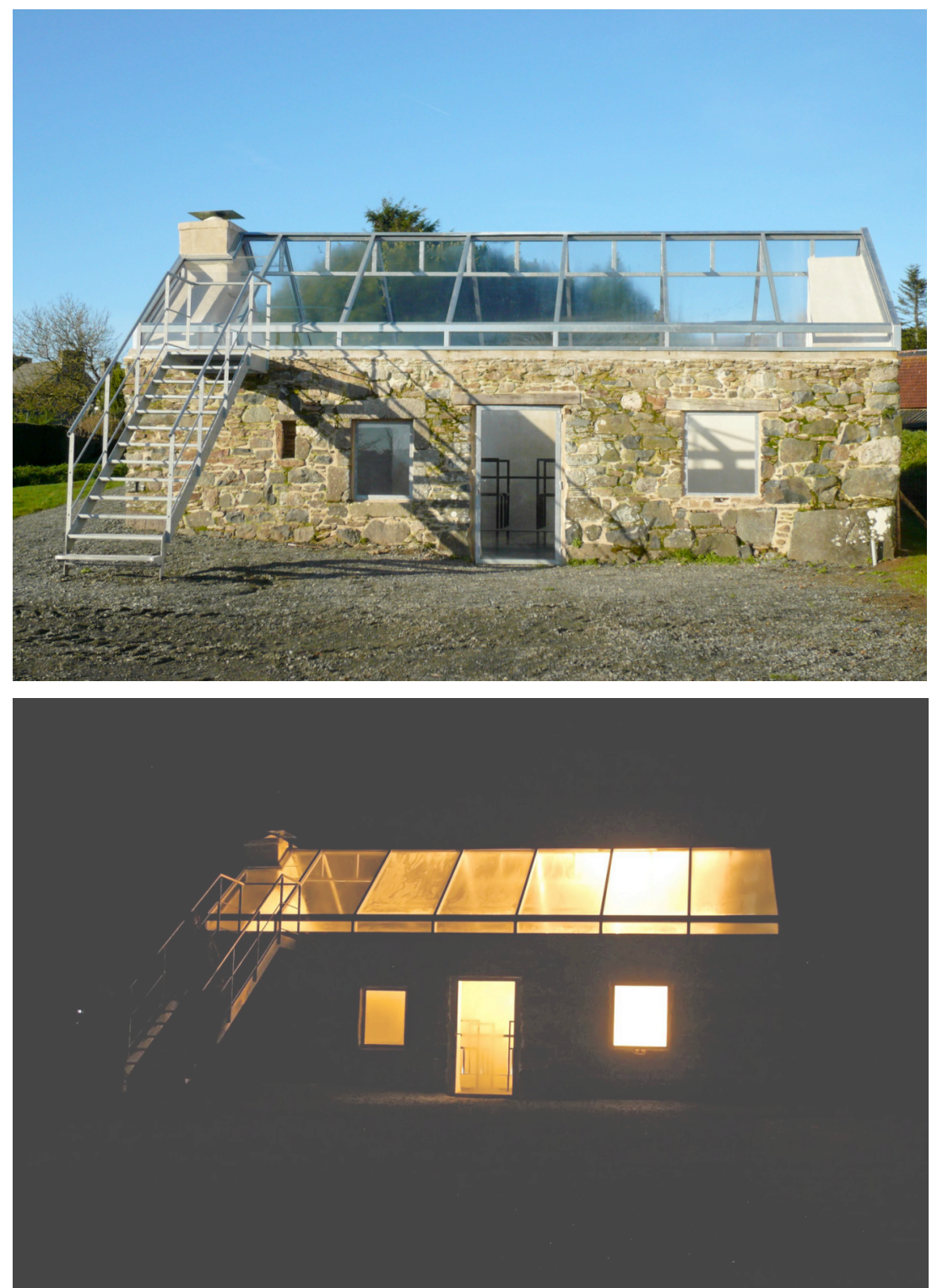

\section{La maison où j'ai grandi à Plougonver}

Commande réalisée pour la Commune de Plougonver (Côtes d'Armor), dans le cadre de l'action Nouveaux commanditaires de la Fondation de France. Médiationproduction : Eternal Network. Caisson de vitrage, escaliers galvanisés, carcasse d'une table et quatre chaises évidées, tubes fluorescents et lampes, projections d'ombres. Photo nuit : Déclic Armor : René Gaudier/ Photo jour : C. Assegond. 